\title{
Waking up to sleep-disordered breathing
}

\section{J A Fleetham}

In 1997 Wright and colleagues ${ }^{1}$ published a systematic review on the health effects of obstructive sleep apnoea syndrome (OSA) and the effectiveness of treatment with continuous positive airway pressure (CPAP). They concluded that there was limited evidence of increased mortality or morbidity in patients with OSA, and that the evidence linking the condition to cardiovascular risk factors and motor vehicle crashes was conflicting and inconclusive. They also concluded that, although CPAP had been shown to improve daytime sleepiness, there were insufficient data to determine its effect on quality of life, morbidity or mortality. This review generated much controversy, but was a wake-up call to investigators in this field that all were not convinced that OSA was an important condition that always warranted treatment. ${ }^{2}$ At that time, our understanding of OSA and the impact of CPAP treatment was at a similar stage to where we were with cardiovascular risk factors such as systemic hypertension, diabetes and hypercholesterolaemia several decades ago. Considerable progress has been made since this review, and many of the issues which it raised have subsequently been addressed in papers published in Thorax.

Systemic hypertension has been reported in epidemiological and hospitalbased studies of patients with OSA, and is independent of obesity and the other common risk factors for hypertension which are also frequently present in this patient population. There have been conflicting data as to whether nasal CPAP can effectively reduce blood pressure in patients with OSA. Hui and associates ${ }^{3}$ performed a randomised controlled study in 56 patients with moderate to severe OSA and daytime sleepiness which showed that 12 weeks of therapeutic CPAP reduced $24 \mathrm{~h}$ mean and diastolic blood pressure by $3.8 \mathrm{mmHg}$ and $3.5 \mathrm{mmHg}$, respectively. No predictors of the change in blood pressure with CPAP

Correspondence to $\mathrm{J}$ A Fleetham, The Lung Centre, Respiratory Division, Vancouver Hospital, 7th Floor, 2775 Laurel Street, Vancouver, BC, Canada V5Z 1M9; john.fleetham@vch.ca treatment have been convincingly identified. Robinson and coworkers ${ }^{4}$ performed a prospective study to determine predictors of blood pressure change. $24 \mathrm{~h}$ blood pressure monitoring was performed on 86 patients with OSA and daytime sleepiness prior to and 6 months after CPAP treatment. The mean $24 \mathrm{~h}$ blood pressure decreased from $101.0 \mathrm{mmHg}$ to $96.1 \mathrm{mmHg}$. The decrease in Epworth sleepiness score and the body mass index were the only significant independent predictors of the decrease in $24 \mathrm{~h}$ blood pressure. Baseline severity of OSA and overnight hypoxaemia were not independent predictors of the decrease in blood pressure. These results suggest that sleep fragmentation and its effects may be more important than hypoxaemia in the pathogenesis of the hypertension associated with OSA.

Type 2 diabetes is a condition of impaired glucose tolerance and insulin resistance which also has a strong causal relationship with central obesity. West and associates ${ }^{5}$ surveyed 1682 men with type 2 diabetes from hospital and primary care practitioner databases. Selected respondents had overnight oximetry to establish whether they had OSA. OSA was highly prevalent in men with type 2 diabetes, most of whom were undiagnosed at the time of the study. Recognition and treatment of OSA in this patient population would be beneficial, especially if they have daytime sleepiness. Excessive daytime sleepiness, obesity and insulin resistance occur frequently in patients with OSA. Ronksley and colleagues ${ }^{6}$ performed a cross-sectional analysis of diabetes and subjective sleepiness in 2149 patients referred for suspected OSA. The prevalence of diabetes increased with greater OSA severity. After correcting for confounding factors, severe OSA was only associated with diabetes in patients with excessive daytime sleepiness. Excessive daytime sleepiness may be a useful clinical marker to identify patients with OSA at risk of metabolic syndrome. ${ }^{7}$ Barcelo and coworkers ${ }^{8}$ examined insulin resistance in 44 patients with OSA with and without excessive daytime sleepiness matched for age, body mass index and severity of OSA and 23 healthy controls. Patients with OSA were re-examined after 3 months of CPAP treatment. Patients with excessive daytime sleepiness had higher plasma levels of glucose and insulin as well as evidence of insulin resistance compared with patients without excessive daytime sleepiness or healthy controls. CPAP treatment reduced insulin resistance in patients with excessive daytime sleepiness but not in those without excessive daytime sleepiness. These findings are analogous to the blood pressure studies described above ${ }^{4}$ whereby the decrease in subjective sleepiness predicted the decline in $24 \mathrm{~h}$ blood pressure with CPAP treatment. West and colleagues ${ }^{9}$ reported a double-blind randomised placebo controlled trial of CPAP treatment in 42 patients with OSA and diabetes over 3 months. Glycaemic control and insulin resistance did not significantly change in either the therapeutic or placebo groups.

Cross and associates ${ }^{10}$ performed a randomised double-blind placebo controlled crossover trial to compare vascular function and examine the impact of CPAP treatment in 46 patients with OSA with and without sleep hypoxaemia. Patients with OSA and sleep hypoxaemia had impaired endothelial vasodilation that was proportional to their hypoxaemia and improved with CPAP therapy. Kohler and colleagues ${ }^{11}$ reported no change in cardiovascular inflammatory markers in a randomised controlled trial of 100 men with moderate to severe OSA treated with CPAP for 4 weeks. These outcomes represent surrogate markers, and the changes in them cannot be extrapolated to infer a reduction in cardiovascular events. The effectiveness of drug treatments such as thrombolysis, aspirin and lipid-lowering treatment in reducing cardiovascular events has been assessed in large long-term randomised controlled trials. ${ }^{12}$ Preliminary results of such a trial of CPAP treatment in patients with OSA have recently been presented. Barbé and colleagues $^{13}$ performed a randomised controlled trial of CPAP versus conservative treatment over 4 years in 724 non-sleepy patients with OSA to evaluate the effect of CPAP treatment on a composite measure of incident cardiovascular events and hypertension. There were 58 cardiovascular events and 148 new cases of hypertension. CPAP treatment significantly reduced the incidence of cardiovascular events and hypertension in those patients who used CPAP for at least $4 \mathrm{~h} /$ night.

The hallmark symptom of OSA is daytime sleepiness, and there is increasing evidence that OSA is associated with an 
increased motor vehicle crash rate. ${ }^{14}$ Mulgrew and associates ${ }^{15}$ compared 3year objective motor vehicle crash rates in 783 patients with suspected OSA and 783 matched control subjects. There were 252 crashes in the patients and 123 crashes in the control subjects. No dose-response effect was observed between the crash rate and OSA severity except in the subgroup of patients in crashes involving personal injury. Furthermore, there was no relationship between crash rate and subjective daytime sleepiness. These findings emphasise the importance of treating all patients with OSA. George ${ }^{16}$ performed a study comparing motor vehicle crash rates for 3 years before and after CPAP treatment in 210 patients with OSA versus 210 matched controls using objective motor vehicle crash records. The crash rate was about three times higher than for control subjects before CPAP treatment and was similar to control subjects after treatment.

Although there is now convincing evidence that CPAP is an effective treatment for OSA, it has still been difficult to convince some healthcare funding agencies to provide funding. Until recently, there were limited data concerning the cost-effectiveness of CPAP treatment. Guest and colleagues ${ }^{17}$ constructed a Markov model including cardiovascular events, motor vehicle crashes and treatment compliance to estimate the cost-effectiveness of CPAP in the management of patients with severe OSA compared with no treatment from the perspective of the UK's National Health Service (NHS). According to the model, $57 \%$ of untreated patients were expected to be alive at the end of 14 years compared with $72 \%$ of patients treated with CPAP. Untreated patients are expected to cost the NHS $£ 10645$ per patient over 14 years compared with $£ 9672$ per CPAP-treated patient. Treatment with CPAP for a period of 1 year was not a cost-effective option since the cost per quality-adjusted life year gained was $>£ 20000$, but after 2 years of treatment the cost per quality-adjusted life year gained was $£ 10000$ or less and, after 13 years of treatment, CPAP was more effective than no treatment for less cost.

Over the last decade patients, physicians and healthcare funding agencies have been waking up to the importance of sleep-disordered breathing. The evidence which independently links OSA to cardiovascular risk factors and motor vehicle crashes is now compelling. CPAP treatment has been shown to decrease some cardiovascular risk factors and motor vehicle crashes. Preliminary results of a large long-term randomised controlled trial indicate that CPAP reduces cardiovascular events and hypertension in patients who comply with treatment. CPAP has been established as a costeffective treatment and many countries now recommend that it should be made available to patients with symptomatic OSA. ${ }^{18} 19$

\section{Competing interests None.}

Provenance and peer review Commissioned; not externally peer reviewed.

Thorax 2010;65:762-763.

doi:10.1136/thx.2010.144931

\section{REFERENCES}

1. Wright J, Johns R, Watt I, et al. Health effects of obstructive sleep apnoea and the effectiveness of continuous positive airway pressure: a systematic review of the research evidence. BMJ 1997:314:851-60.

2. Fleetham JA. A wake up call for sleep disordered breathing. BMJ 1997;314:839-40.

3. Hui DS, To KW, Ko FW, et al. Nasal CPAP reduces systemic blood pressure in patients with obstructive sleep apnoea and mild sleepiness. Thorax 2006;61:1083-90.

4. Robinson GV, Langford BA, Smith DM, et al. Predictors of blood pressure fall with continuous positive airway pressure (CPAP) treatment of obstructive sleep apnoea (OSA). Thorax 2008:63:855-9.
5. West SD, Nicoll DJ, Stradling JR. Prevalence of obstructive sleep apnoea in men with type 2 diabetes. Thorax 2006;61:945-50.

6. Ronksley PE, Hemmelgarn BR, Heitman SJ, et al. Obstructive sleep apnoea is associated with diabetes in sleepy subjects. Thorax 2009;64:834-9.

7. Ip M. Obstructive sleep apnoea, insulin resistance and sleepiness. Thorax 2008;63:939-40.

8. Barceló A, Barbé $F$, de la Peña $M$, et al. Insulin resistance and daytime sleepiness in patients with sleep apnoea. Thorax 2008;63:946-50.

9. West SD, Nicoll DJ, Wallace TM, et al. Effect of CPAP on insulin resistance and $\mathrm{HbA} 1 \mathrm{c}$ in men with obstructive sleep apnoea and type 2 diabetes. Thorax 2007:62:969-74.

10. Cross MD, Mills NL, Al-Abri M, et al. Continuous positive airway pressure improves vascular function in obstructive sleep apnoea/hypopnoea syndrome: a randomised controlled trial. Thorax 2008;63:578-83.

11. Kohler M, Ayers L, Pepperell JCT, et al. Effects of continuous positive airway pressure on systemic inflammation in patients with moderate to severe obstructive sleep apnoea: a randomised controlled trial. Thorax 2009;64:67-73.

12. Ayas NT, Mancini GBJ, Fleetham JA. Does CPAP delay the development of cardiovascular disease in patients with obstructive sleep apnoea hypopnoea? Thorax 2006;61:459-60.

13. Barbé $\mathbf{F}$, Duran-Cantolla J, Carmona $\mathrm{C}$, et al. Effect of CPAP treatment on the incidence of cardiovascular events and hypertension in non-sleepy OSAS patients. A long-term RCT. Am J Respir Crit Care Med 2010:181:A5559.

14. Stradling J. Driving and obstructive sleep apnoea. Thorax 2008;63:481-3.

15. Mulgrew AT, Nasvadi G, Butt A, et al. Risk and severity of motor vehicle crashes in patients with obstructive sleep apnea hypopnea. Thorax 2008;63:536-41.

16. George CF. Reduction in motor vehicle collisions following treatment of sleep apnoea with nasal CPAP. Thorax 2001:56:508-12.

17. Guest JF, Helter MT, Morga A, et al. Costeffectiveness of using continuous positive airway pressure in the treatment of severe obstructive sleep apnoea/hypopnoea syndrome in the UK. Thorax 2008;63:860-5

18. National Institute for Health and Clinical

Excellence. Continuous positive airway pressure for the treatment of obstructive sleep apnoea/hypopnoea syndrome. 2007. http://www.nice.org.uk/guidance/ index.jsp?action $=$ bylD\&0 $=11944$.

19. Centers for Medicare and Medicaid Services. Decision memo for continuous positive airway pressure (CPAP) therapy for obstructive sleep apnea (OSA) (CAG-00093R2). https://www.cms.hhs.gov/ $\mathrm{mcd} /$ viewdecisionmemo.asp?

from2 $=$ viewdecisionmemo.asp\&id $=204 \varepsilon$

\section{Travelling in time with COPD}

\section{J R Hurst, ${ }^{1}$ P M A Calverley ${ }^{2}$}

${ }^{1}$ Academic Unit of Respiratory Medicine, UCL Medical School, London, UK; ${ }^{2}$ Clinical Science Centre, University Hospital Aintree, Liverpool, UK

Correspondence to J R Hurst, Academic Unit of Respiratory Medicine, UCL Medical School, London, UK; j.hurst@medsch.ucl.ac.uk
In the last 8 years the $\mathrm{FEV}_{1}$ (forced expiratory volume in $1 \mathrm{~s}$ ) of a 'typical' patient with chronic obstructive pulmonary disease (COPD) has fallen by $\sim 400 \mathrm{ml}$. However, the knowledge of those caring for this hypothetical patient has grown rapidly in this same period and Thorax has played a vital role in this change of fortune. In 2003 there were 1698 citations to COPD articles in PubMed, compared with 1494 in the first 6 months of 2010 alone, a remarkable growth in the interest in and perhaps quality of research in this field.

Many themes have been considered in this last 8 years, not least how to diagnose the disease. Swanney et $a l^{1}$ defined population norms for the lower limit of normal of the $\mathrm{FEV}_{1} /$ forced vital capacity (FVC) 